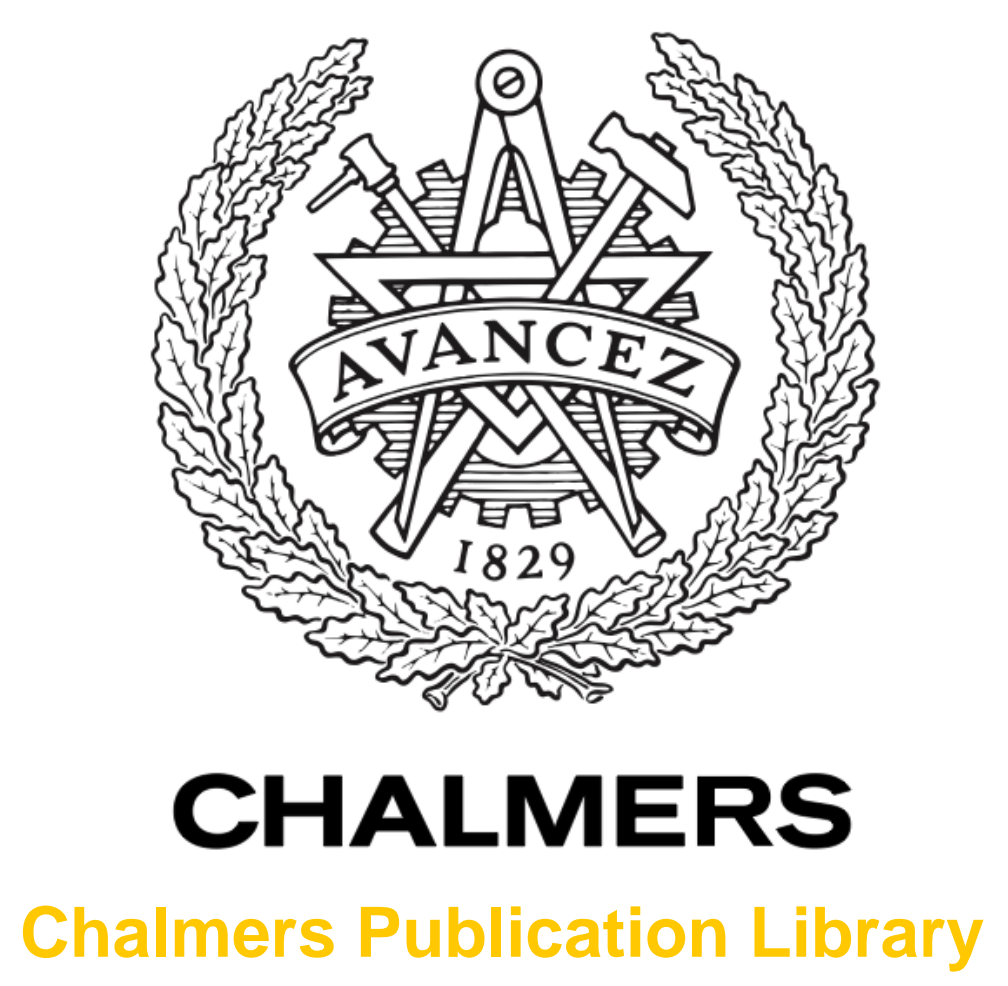

\title{
Performance analysis of distributed MIMO systems in Rayleigh/Inverse- Gaussian fading channels
}

This document has been downloaded from Chalmers Publication Library (CPL). It is the author's version of a work that was accepted for publication in:

IEEE Global Communications Conference, GLOBECOM 2012, Anaheim, United States, 3-7 December 2012 (ISSN: 1930-529X)

Citation for the published paper:

Gopal, V. ; Matthaiou, M. ; Zhong, C. (2012) "Performance analysis of distributed MIMO systems in Rayleigh/Inverse- Gaussian fading channels". IEEE Global Communications Conference, GLOBECOM 2012, Anaheim, United States, 3-7 December 2012 pp. 24682474.

http://dx.doi.org/10.1109/GLOCOM.2012.6503487

Downloaded from: http://publications.lib.chalmers.se/publication/177662

Notice: Changes introduced as a result of publishing processes such as copy-editing and formatting may not be reflected in this document. For a definitive version of this work, please refer to the published source. Please note that access to the published version might require a subscription. 


\title{
Performance Analysis of Distributed MIMO Systems in Rayleigh/Inverse-Gaussian Fading Channels
}

\author{
Vetriselvam Gopal*, Michail Matthaiou*, and Caijun Zhong ${ }^{\dagger}$ \\ *Department of Signals and Systems, Chalmers University of Technology, Gothenburg, Sweden \\ $\dagger$ Institute of Information and Communication Engineering, Zhejiang University, Hangzhou, China \\ E-mail: gopalv@student.chalmers.se,michail.matthaiou@chalmers.se, caijunzhong@zju.edu.cn
}

\begin{abstract}
In this paper, we pursue a performance evaluation of distributed multiple-input multiple-output (MIMO) systems in composite Rayleigh/Inverse-Gaussian fading channels. Capitalizing on some generic bounding techniques, we first derive new closed-form bounds on the ergodic capacity of optimal receivers. In order to gain useful insights into the impact of fading parameters on optimal receivers' performance, a detailed characterization in the asymptotically high and low signal-tonoise ratio regimes is also provided. In addition, we explore the "large-system" regime and provide asymptotic expressions when the number of antennas grows large. A similar performance analysis is performed for the achievable sum rate of distributed MIMO systems employing linear minimum mean-square error receivers.
\end{abstract}

\section{INTRODUCTION}

The potential of combining MIMO spatial multiplexing gains with macro-diversity gains is realized by distributed MIMO (D-MIMO) systems, that promise to improve the cell coverage [1]-[3]. In D-MIMO systems, multiple antennas, placed at one end of the radio link, are deployed into multiple radio ports. In such configurations, each radio port experiences different large-scale fading (a.k.a. shadowing) effects and pathloss, due to the different propagation paths. This is the key difference compared to conventional point-to-point MIMO configurations, which makes the performance analysis of DMIMO systems a challenging mathematical problem. As such, there are very few analytical works investigating the impact of composite fading channels (i.e., mixture of both smallscale and large-scale fading) on the performance of D-MIMO systems.

The ergodic capacity of D-MIMO systems was explored with the aid of majorization theory in [2] by deriving upper and lower capacity bounds for the case of Nakagami- $m / \log$-normal fading channels. On the other hand, in [3], the analytically friendlier gamma distribution was used, as an alternative to the log-normal distribution, and the capacity of D-MIMO systems was investigated over Nakagami- $m /$ Gamma fading (a.k.a. generalized- $\mathcal{K}$ fading) channels. Nevertheless, these results, based on the gamma shadowing model, are essentially approximations. More importantly, for the large variance case or in the tails of the log-normal distribution, the gamma distribution does not yield a good approximation [4]. Motivated by these intrinsic deficiencies, the Inverse-Gaussian (IG) distribution was recently proposed as a more accurate approximation to the classical log-normal model to describe large- scale fading effects [4]. In fact, the authors in [4] proved that the composite Rayleigh/IG (RIG) distribution approximates the Rayleigh/log-normal distribution more accurately than the $\mathcal{K}$ distribution in terms of the Kullback-Leibler measure of divergence. Note that the IG distribution has already been used in the context of free-space optical systems [5], whereas the composite Nakagami- $m / \mathrm{IG}$ distribution was first used in [6] for the performance evaluation of communications systems and in [7], in the context of relaying systems, respectively.

The main objective of this paper is to analytically investigate the performance of D-MIMO systems with optimal and linear minimum mean-squared error (MMSE) receivers over RIG fading channels. It is important to note that, to the best of authors' knowledge, the results in this paper present the first-ever analytical investigation of D-MIMO systems in RIG fading channels. Motivated by the preceding, we hereafter derive tight upper and lower sum rate bounds for this class of channels using some recent bounding techniques [2]. With the help of these proposed bounds, we analytically explore the "largesystem" regime by assuming that either the number of receive or transmit antennas grows large. In both cases, we explicitly demonstrate that the effects of small-scale Rayleigh fading are averaged out and the ergodic capacity is affected only by the large-scale fading. We also investigate the asymptotically high and low signal-to-noise ratio (SNR) regimes. Our analytical results are quite informative and insightful; for example, they enable us to characterize the impact of large-scale fading parameters as well as path-loss on the sum rate.

Notation: For any matrix, $(\cdot)^{H}$ denotes the Hermitian transpose, $\operatorname{tr}(\cdot)$ the matrix trace, and $\operatorname{det}(\cdot)$ the determinant operation. The $(i, j)$-th minor of matrix $\mathbf{A}$ is denoted by $\mathbf{A}_{i j}$, while $\mathbf{A}_{i}$ is $\mathbf{A}$ with the $i$-th column removed. The symbol $\mathcal{E}[\cdot]$ stands for the expectation operation. The notation $\Gamma(\cdot)$ stands for the well-known Gamma function [8, Eq. (8.310.1)], while $\operatorname{Ei}(x)=-\int_{-x}^{\infty} \frac{e^{-t}}{t} d t$ is the exponential integral function [8, Eq. (8.211.1)]. Finally, $\psi(\cdot)$ is Euler's digamma function [8, Eq. (8.360.1)].

\section{MIMO System MOdEL}

Consider a typical MIMO system with $N_{r}$ receive antennas and $L$ radio ports each connected to $N_{t}$ transmit antennas, assuming that $N_{r} \geq L N_{t}$. The input-output model for this D-MIMO system is

$$
\mathbf{y}=\sqrt{\gamma} \mathbf{H} \mathbf{\Xi}^{1 / 2} \mathbf{x}+\mathbf{n}
$$


where $\mathbf{x} \in \mathbb{C}^{L N_{t} \times 1}$ and $\mathbf{y} \in \mathbb{C}^{N_{r} \times 1}$ are the transmitted and received signal vectors, respectively, while $\mathbf{n} \sim \mathcal{C N}\left(\mathbf{0}, N_{0} \mathbf{I}_{N_{r}}\right)$ is the complex AWGN term, where $N_{0}$ is the noise power. The average SNR is defined as $\gamma$. The large-scale fading effects are represented by the diagonal matrix $\boldsymbol{\Xi} \in \mathbb{R}^{L N_{t} \times L N_{t}}$ whose structure is $\boldsymbol{\Xi}=\operatorname{diag}\left\{\mathbf{I}_{N_{t}} \xi_{i} / D_{i}^{v}\right\}$ for $i=1, \ldots, L$. Note that all $N_{t}$ antennas in the $i$-th radio port experience the same large-scale fading. The distance between the receiver and the $i$-th radio port is denoted by $D_{i}$, while $v$ is the path-loss exponent. The large-scale fading coefficients $\xi_{i}$ are modeled as independent IG random variables $(\mathrm{RVs}), \xi_{i} \sim \operatorname{IG}\left(\mu_{i}, \lambda_{i}\right)$, or

$$
p\left(\xi_{i}\right)=\sqrt{\frac{\lambda_{i}}{2 \pi}} \xi_{i}^{-\frac{3}{2}} \exp \left(-\frac{\lambda_{i}\left(\xi_{i}-\mu_{i}\right)^{2}}{2 \mu_{i}^{2} \xi_{i}}\right), \quad \xi_{i}>0
$$

where $\mu_{i}>0, \lambda_{i}>0$ are the mean and the scale parameters of the IG distribution, respectively [9].

The entries of the channel matrix $\mathbf{H} \in \mathbb{C}^{N_{r} \times L N_{t}}$ are assumed to be independent and identically distributed (i.i.d.) Rayleigh RVs, i.e., $h_{s t} \sim \mathcal{C N}(0,1)$, where $s=1, \ldots, N_{r}$ and $t=1, \ldots, L N_{t}$. We now invoke that a squared Rayleigh RV follows the Gamma distribution with scale parameter 1 and also that the sum of $n$ i.i.d. Gamma RVs with common scale parameter $\theta$ and shape parameters $\left\{k_{i}\right\}_{i=1}^{n}$ is also Gamma distributed with parameters $\left(\sum_{i=1}^{n} k_{i}, \theta\right)$ [10]. As such, the sum of $N_{r}$ i.i.d. Gamma RVs with scale parameter 1 is distributed as $\varphi_{i} \sim \operatorname{Gamma}\left(N_{r}, 1\right)$, or

$$
p\left(\varphi_{i}\right)=\frac{\varphi_{i}^{N_{r}-1}}{\Gamma\left(N_{r}\right)} \exp \left(-\varphi_{i}\right), \quad \varphi_{i} \geq 0 .
$$

\section{Performance analysis of D-MiMO systems}

In this section, we present a detailed performance analysis of D-MIMO systems with optimal and linear MMSE receivers in RIG fading channels.

\section{A. Optimal receivers}

We assume that the receiver has perfect channel state information (CSI) while the transmitter has nor statistical neither instantaneous CSI and as such performs uniform power allocation across all the data streams. Then, the MIMO ergodic capacity reads as

$$
\mathcal{C}_{\text {erg }}=\mathcal{E}\left[\log _{2}\left(\operatorname{det}\left(\mathbf{I}_{L N_{t}}+\frac{\gamma}{L N_{t}} \boldsymbol{\Xi} \mathbf{H}^{H} \mathbf{H}\right)\right)\right]
$$

where the expectation is taken over all channel realizations of $\mathbf{H}, \boldsymbol{\Xi}$ and the channel is assumed to be ergodic.

1) Exact analysis: We now derive new ergodic capacity upper and lower bounds for optimal receivers. Capitalizing on the results of [2], we first derive an upper capacity bound.

Proposition 1: For D-MIMO systems with optimal receivers in RIG fading channels, the ergodic capacity in (4) is upper bounded by $\mathcal{C}_{\mathrm{UB}}$, with

$$
\mathcal{C}_{\mathrm{UB}}=\frac{\gamma}{2 \sqrt{\pi} \ln 2 L \Gamma\left(N_{r}\right)} \sum_{i=1}^{L} \frac{1}{D_{i}^{v}} \exp \left(\frac{\lambda_{i}}{\mu_{i}}\right) \sum_{j=1}^{N} w_{j} V_{i}\left(x_{j}\right)
$$

where $V_{i}(t)=t^{-\frac{1}{2}} \exp \left(-\frac{\lambda_{i}^{2}}{4 \mu_{i}^{2} t}\right) G_{3,2}^{1,3}\left(\left.\frac{\gamma_{i} \mu_{i} t}{L N_{t} \lambda_{i} D_{i}^{v}}\right|_{0,-1} ^{-N_{r}, 0,0}\right)$, $\left\{x_{j}\right\}_{j=1}^{N}$ are the zeros of the $N$-th order Laguerre polynomial, $\left\{w_{j}\right\}_{j=1}^{N}$ are the weight factors tabulated in [11, Table 25.9], and $G_{p, q}^{m, n}=\left[\left.x\right|_{a_{1}, \ldots, a_{p}} ^{b_{1}, \ldots, b_{q}}\right]$ denotes the Meijer's- $G$ function [8, Eq. (9.301)].

Proof: Using [2, Eq. (64)], we can rewrite the ergodic capacity in (4) as

$$
\mathcal{C}_{\text {erg }} \leq \mathcal{C}_{\mathrm{UB}}=\frac{N_{t}}{\ln 2} \sum_{i=1}^{L} \underbrace{\mathcal{E}\left[\ln \left(1+\frac{\gamma}{L N_{t}} \frac{\xi_{i} \varphi_{i}}{D_{i}^{v}}\right)\right]}_{I_{1}} .
$$

To evaluate $I_{1}$ we first express $\ln (1+a x)$ in terms of a Meijer's- $G$ function using [12, Eq. (8.4.6.5)]. Then, combining (3) with $[8$, Eq. (7.813.1)] gives

$$
I_{1}=\frac{1}{\Gamma\left(N_{r}\right)} \int_{0}^{\infty} G_{3,2}^{1,3}\left(\left.\frac{\gamma_{i} \xi_{i}}{L N_{t} D_{i}^{v}}\right|_{1,0} ^{1-N_{r}, 1,1}\right) p\left(\xi_{i}\right) d \xi_{i} .
$$

Substituting (2) into (7), applying a change of variables, $t_{i}=$ $\left(2 \xi_{i} \mu_{i}^{2} / \lambda_{i}\right)$, and thereafter using [8, Eq. (9.31.5)], we end up with the following expression

$$
I_{1}=\frac{\gamma \exp \left(\frac{\lambda_{i}}{\mu_{i}}\right)}{2 \sqrt{\pi} L N_{t} D_{i}^{v} \Gamma\left(N_{r}\right)} \int_{0}^{\infty} \exp \left(-t_{i}\right) V_{i}\left(t_{i}\right) d t_{i}
$$

where $V_{i}\left(t_{i}\right)$ is defined in (5). The above integral can be efficiently evaluated by Gauss-Laguerre quadratic integration [11, Eq. (25.4.45)]. Thus, we can conclude the proof.

In the above proof, Gauss-Laguerre quadratic integration has been used to approximate the integral expression. While (5) can be used to compute the upper bound for D-MIMO systems in RIG fading channels, the computation of GaussLaguerre quadratic integration can still be time consuming, especially at low SNRs (e.g., $\gamma<-15 \mathrm{~dB}$ ). More importantly, the above upper bound, though in analytical form, provides limited physical insights. It is thus of interest to consider the high-SNR regime for further analyzing the upper bound.

Corollary 1: At high-SNRs $(\gamma \rightarrow \infty)$, the ergodic capacity upper bound $\mathcal{C}_{\mathrm{UB}}$ simplifies to

$$
\begin{aligned}
\mathcal{C}_{\mathrm{UB}}^{\infty} & =L N_{t} \log _{2}\left(\frac{\gamma}{L N_{t}}\right)+\frac{L N_{t}}{\ln 2} \psi\left(N_{r}\right)+N_{t} \sum_{i=1}^{L}\left(\log _{2}\left(\mu_{i}\right)\right. \\
& \left.-v \log _{2}\left(D_{i}\right)+\frac{1}{\ln 2} \exp \left(\frac{2 \lambda_{i}}{\mu_{i}}\right) \operatorname{Ei}\left(\frac{-2 \lambda_{i}}{\mu_{i}}\right)\right)
\end{aligned}
$$

Proof: The proof is trivial and therefore omitted.

Clearly, the high-SNR upper bound in (9) effectively decouples the effects of small-scale and large-scale fading on the ergodic capacity. Now, we give a new ergodic capacity lower bound via the following proposition:

Proposition 2: For D-MIMO systems with optimal receivers in RIG fading channels, the ergodic capacity in (4) 
is lower bounded by $\mathcal{C}_{\mathrm{LB}}$, with

$$
\begin{aligned}
& \mathcal{C}_{\mathrm{LB}}=L N_{t} \log _{2}\left(1+\frac{\gamma}{L N_{t}} \exp \left(\frac { 1 } { L } \sum _ { i = 1 } ^ { L } \left(\ln \mu_{i}-v \ln D_{i}\right.\right.\right. \\
& \left.\left.\left.+\exp \left(\frac{2 \lambda_{i}}{\mu_{i}}\right) \operatorname{Ei}\left(\frac{-2 \lambda_{i}}{\mu_{i}}\right)\right)+\frac{1}{L N_{t}} \sum_{k=0}^{L N_{t}-1} \psi\left(N_{r}-k\right)\right)\right) .
\end{aligned}
$$

Proof: The proof relies on the application of Minkowski's inequality to (4), as proposed in [13, Th. 1]. Exploiting the fact that $\ln (1+\alpha \exp (x))$ is convex in $x$ for $\alpha>0$, and thereafter applying Jensen's inequality, we can obtain the following lower bound

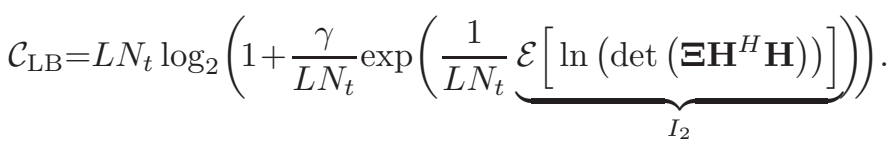

Recalling the identity $\operatorname{det}(\mathbf{A B})=\operatorname{det}(\mathbf{A}) \operatorname{det}(\mathbf{B})$, we can express the above expectation term as follows:

$$
I_{2}=\mathcal{E}[\ln (\operatorname{det}(\boldsymbol{\Xi}))]+\mathcal{E}\left[\ln \left(\operatorname{det}\left(\mathbf{H}^{H} \mathbf{H}\right)\right)\right] .
$$

Since $\Xi$ is diagonal, the first term in (11) can be given as

$$
\begin{aligned}
& \mathcal{E}[\ln (\operatorname{det}(\boldsymbol{\Xi}))] \stackrel{(50)}{=}-N_{t} v \sum_{i=1}^{L} \ln D_{i} \\
& \quad+N_{t} \sum_{i=1}^{L}\left(\ln \mu_{i}+\exp \left(\frac{2 \lambda_{i}}{\mu_{i}}\right) \operatorname{Ei}\left(\frac{-2 \lambda_{i}}{\mu_{i}}\right)\right) .
\end{aligned}
$$

Since $\mathbf{H}$ is Rayleigh distributed, the term $\mathbf{H}^{H} \mathbf{H}$ follows a central Wishart distribution (zero-mean) [14]. Using [14, Eq. (A.8.1)], the last term in (11) can be easily evaluated. Thus, we can conclude the proof.

Note that, in the high-SNR regime, the lower bound becomes by definition exact and equal to the ergodic capacity [13].

2) Low-SNR analysis: We now examine the ergodic capacity in the power-limited (or low-SNR) regime. The low-SNR performance of MIMO systems is typically analyzed via the normalized receive energy per bit $\left(E_{b}^{r} / N_{0}\right)$ rather than via the per-symbol SNR [15]. This capacity representation is given by

$$
\begin{gathered}
\mathcal{C}_{\text {erg }}\left(\frac{E_{b}}{N_{0}}\right) \approx \mathcal{S}_{0} \log _{2}\left(\frac{\frac{E_{b}}{N_{0}}}{\frac{E_{b}}{N_{0} \text { min }}}\right) \\
\frac{E_{b}}{N_{0 \text { min }}}=\frac{1}{\dot{\mathcal{C}}_{\text {erg }}(0)} \text { and } \mathcal{S}_{0}=-2 \ln 2 \frac{\left(\dot{\mathcal{C}}_{\mathrm{erg}}(0)\right)^{2}}{\ddot{\mathcal{C}}_{\text {erg }}(0)}
\end{gathered}
$$

where $E_{b} / N_{0_{\min }}$ and $\mathcal{S}_{0}$ are the minimum normalized energy per information bit required to convey any positive rate reliably and the wideband slope, respectively, while $\dot{\mathcal{C}}_{\text {erg }}(0)$ and $\ddot{\mathcal{C}}_{\text {erg }}(0)$ denote the first- and second-order derivatives of the ergodic capacity in (4) w.r.t. the SNR, respectively [15].

Proposition 3: For D-MIMO systems with optimal receivers in RIG fading channels, the minimum energy per information bit and the wideband slope are

$$
\begin{aligned}
{\frac{E_{b}}{N_{0 \text { min }}}}^{\text {opt }}=\frac{L \ln 2}{N_{r}}\left(\sum_{i=1}^{L} \mu_{i} D_{i}^{-v}\right)^{-1} \\
\mathcal{S}_{0}^{\text {opt }}=\frac{2}{\frac{1}{N_{r}}+\frac{1}{N_{t}}\left(\frac{\sum_{i=1}^{L}\left(\mu_{i}^{2}+\frac{\mu_{i}^{3}}{\lambda_{i}}\right) D_{i}^{-2 v}}{\left(\sum_{i=1}^{L} \mu_{i} D_{i}^{-v}\right)^{2}}\right)} .
\end{aligned}
$$

Proof: First, we recall that

$$
\left.\frac{d}{d x} \ln (\operatorname{det}(\mathbf{I}+x \mathbf{A}))\right|_{x=0}=\operatorname{tr}(\mathbf{A}) .
$$

Now, we set $\gamma \rightarrow 0$ in (6) and evaluate $\dot{\mathcal{C}}_{\text {erg }}$ as

$$
\begin{aligned}
\dot{\mathcal{C}}_{\mathrm{erg}}(0) & =\mathcal{E}\left[\operatorname{tr}\left(\mathbf{Z}^{H} \mathbf{Z}\right)\right] \\
& =\frac{N_{t}}{L N_{t} \ln 2} \sum_{i=1}^{L} \int_{0}^{\infty} \int_{0}^{\infty} \frac{\xi_{i} \varphi_{i}}{D_{i}^{v}} p\left(\varphi_{i}\right) p\left(\xi_{i}\right) d \varphi_{i} d \xi_{i}
\end{aligned}
$$

where $\mathbf{Z}=\mathbf{H} \boldsymbol{\Xi}^{1 / 2}$ and $\xi_{i} \varphi_{i} / D_{i}^{v}$ are the real, non-negative diagonal elements of $\mathbf{Z}^{H} \mathbf{Z}$. Substituting (2) and (3) into (18), thereafter using [8, Eq. (3.381.4)] and the first moment of a IG RV $\left(\mathcal{E}\left[\xi_{i}\right]=\mu_{i}\right)$, we get

$$
\dot{\mathcal{C}}_{\text {erg }}(0)=\frac{N_{r}}{L \ln 2} \sum_{i=1}^{L} \mu_{i} D_{i}^{-v}
$$

For the wideband slope $\mathcal{S}_{0}^{\text {opt }}$, we invoke a classical result from random matrix theory on correlated Rayleigh MIMO channels [16, Eq. (19)]. We assume no correlation at the receiver (i.e., $\Theta_{R}=\mathbf{I}_{N_{r}}$ and $\boldsymbol{\Theta}_{T}=\Xi$ ) and we can obtain the dispersion of random matrix $\Xi$ [16, Eq. (8)] using (51) and (52). Thus, we can conclude the proof.

Note that $E_{b} / N_{0_{\min }}^{\text {opt }}$ in (15) is independent of $N_{t}$, which agrees with the results of [15] and [16], while a higher $N_{r}$ improves the low-SNR capacity in (13) by reducing $E_{b} / N_{0_{\text {min }}}^{\text {opt }}$. On the other hand, the presence of the large-scale fading mean parameter $\mu_{i}$ increases $E_{b} / N_{0_{\text {min }}}^{\text {opt }}$, especially in severe fading conditions (i.e., small values of $\mu_{i}$ ). Note that the wideband slope in (16) is by definition always greater than one.

We can now validate the above theoretical expressions via a set of Monte-Carlo simulations. We generate 10,000 random realizations of the small- and large-scale fading matrices, $\mathbf{H}$ and $\Xi$, respectively. In Fig. 1, the simulated ergodic capacity of (4) is compared with the analytical low-SNR approximation in (13). We change only the large-scale fading mean parameter $\mu_{i}$ and keep all other fading parameters constant. We can clearly observe that the low-SNR capacity increases, whereas the wideband slope decreases when $\mu_{i}$ gets larger. On the other hand, the analytical approximation becomes tighter for smaller values of $\mu_{i}$.

3) High-SNR analysis: We now examine the ergodic capacity in the high-SNR $(\gamma \rightarrow \infty)$ regime. To get better insights into the high-SNR capacity performance, we can invoke the following affine capacity expansion, which was originally 


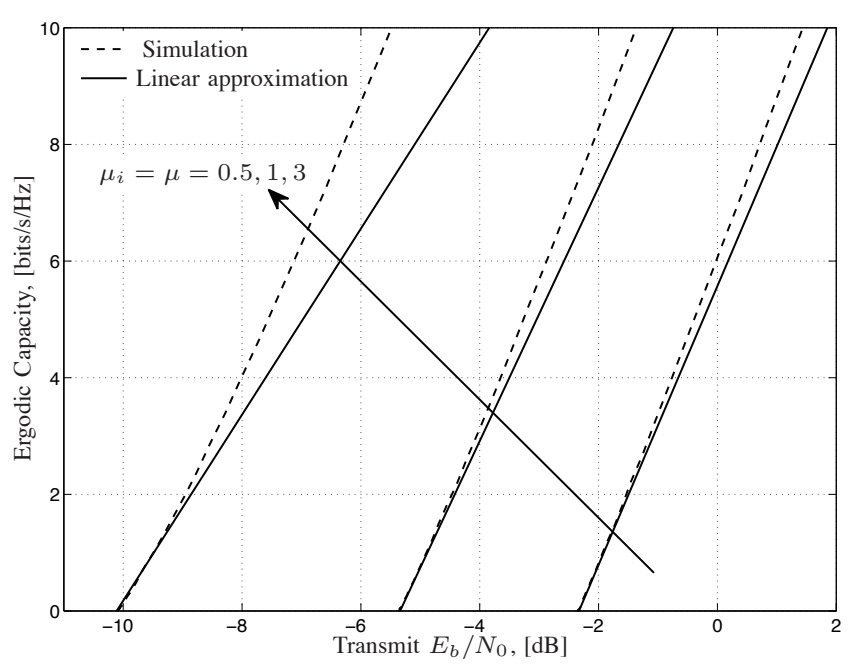

Fig. 1. Simulated ergodic capacity and low-SNR approximation against the transmit $E_{b} / N_{0}$ for different values of the large-scale fading mean $\mu_{i}=\mu$ parameter $\left(N_{r}=12, N_{t}=2, L=3, \lambda_{i}=\lambda=3, D_{i}=D=1500 \mathrm{~m}\right.$ $(\forall i=1, \ldots, L)$, and $v=4)$.

applied in the context of multiple access systems with random spreading [17] and thereafter in the analysis of MIMO systems [18]:

$$
\mathcal{C}_{\text {erg }}=\mathcal{S}_{\infty}\left(\log _{2}(\gamma)-\mathcal{L}_{\infty}\right)+o(1)
$$

where $\mathcal{S}_{\infty}$ is the so-called high-SNR slope in bits/s/Hz per $3-\mathrm{dB}$ units, while $\mathcal{L}_{\infty}$ is the zero-th order term or high-SNR power offset, in 3-dB units [17], [18].

Proposition 4: For D-MIMO systems with optimal receivers in RIG fading channels, the high-SNR parameters are

$$
\begin{aligned}
& \mathcal{S}_{\infty}^{\mathrm{opt}}=L N_{t} \\
& \mathcal{L}_{\infty}^{\mathrm{opt}}=\log _{2}\left(L N_{t}\right)-\frac{1}{L} \sum_{i=1}^{L}\left(\log _{2}\left(\mu_{i}\right)-v \log _{2}\left(D_{i}\right)\right. \\
&\left.+\frac{1}{\ln 2} \exp \left(\frac{2 \lambda_{i}}{\mu_{i}}\right) \operatorname{Ei}\left(\frac{-2 \lambda_{i}}{\mu_{i}}\right)\right)-\frac{1}{L N_{t} \ln 2} \sum_{k=0}^{L N_{t}-1} \psi\left(N_{r}-k\right) .
\end{aligned}
$$

Proof: For MIMO systems with optimal receivers, the slope and the offset are obtained by [19, Eq. (16), (17)]

$$
\begin{aligned}
& \mathcal{S}_{\infty}^{\text {opt }}=\min \left(N_{r}, L N_{t}\right) \\
& \mathcal{L}_{\infty}^{\text {opt }}=\log _{2}\left(L N_{t}\right)-\frac{1}{L N_{t} \ln 2} \mathcal{E}\left[\ln \left(\operatorname{det}\left(\boldsymbol{\Xi} \mathbf{H}^{H} \mathbf{H}\right)\right)\right] .
\end{aligned}
$$

Combining (11) with (24) gives (22) after simplifications.

From (22), we can infer that the small- and large-scale fading terms are effectively decoupled in the high-SNR regime. Furthermore, larger values of $D_{i}$, reduce the ergodic capacity due to the increased path-loss attenuation. Note that the highSNR slope in (21) verifies that the high-SNR ergodic capacity increases linearly with the minimum number of antennas, which is in line with [13] and [14].

In Fig. 2, the simulated ergodic capacity is compared with the analytical high-SNR approximation (20), the analytical upper and lower bounds from (5) and (10), respectively.

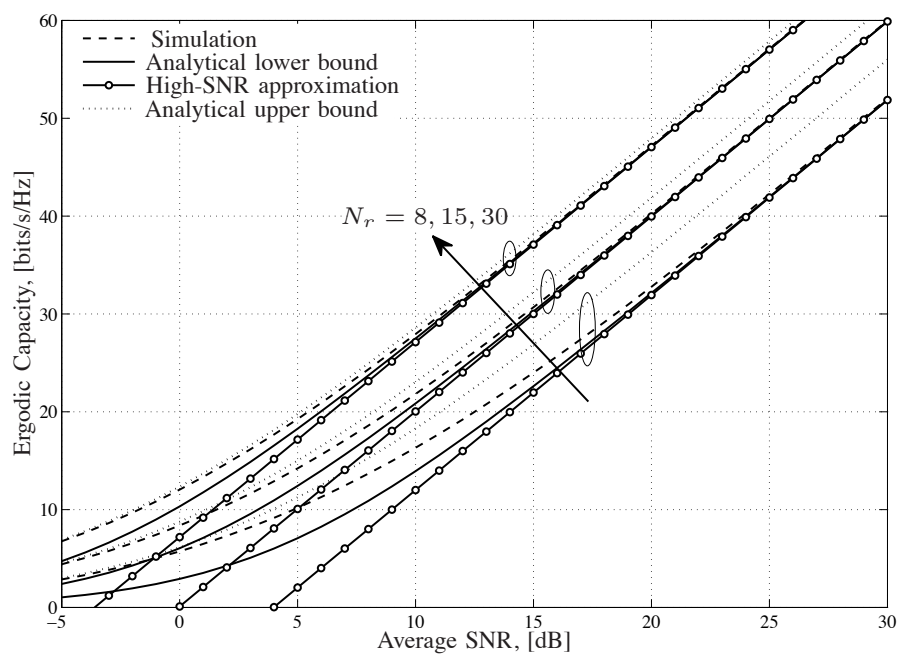

Fig. 2. Simulated ergodic capacity, analytical high-SNR approximation, analytical upper and lower bounds against the average $\operatorname{SNR}\left(N_{t}=2, L=3\right.$, $\mu_{i}=[4,2,3], \lambda_{i}=[3,5,7], D_{i}=[1000 \mathrm{~m}, 1500 \mathrm{~m}, 2000 \mathrm{~m}]$, where $i=1, \ldots, L$, and $v=4)$.

Both the upper and lower bounds become tighter when the number of receive antennas, $N_{r}$, increases. Also, we can easily observe that the high-SNR approximations become exact even at moderate SNR values. In the low-SNR regime, the lower bound converges asymptotically to the empirical value of ergodic capacity. These observations are consistent with the results of [3], [13], and [20].

4) Large-system analysis: We now examine the ergodic capacity lower bound in the large-system regime.

Corollary 2: When the number of receive antennas grows large (i.e., $N_{r} \rightarrow \infty$ ), while $N_{t}, L$ are kept fixed, the ergodic capacity lower bound becomes

$$
\begin{aligned}
\mathcal{C}_{\mathrm{LB}} & =L N_{t} \log _{2}\left(1+\frac{\gamma N_{r}}{L N_{t}} \exp \left(\frac { 1 } { L } \sum _ { i = 1 } ^ { L } \left(\ln \mu_{i}-v \ln D_{i}\right.\right.\right. \\
& \left.\left.\left.+\exp \left(\frac{2 \lambda_{i}}{\mu_{i}}\right) \operatorname{Ei}\left(\frac{-2 \lambda_{i}}{\mu_{i}}\right)\right)\right)\right) .
\end{aligned}
$$

Proof: First, we recall that [11, Eq. (6.3.18)]

$$
\psi(x) \approx \ln x \text {, if } x \rightarrow \infty .
$$

Substituting (26) into (10) gives (25) after appropriate simplifications.

From the large-system result in (25), we can clearly conclude that the small-scale fading effects are asymptotically averaged out and only the large-scale fading effects remains, when the number of receive antennas grows very large, which agrees with the results of [21]. Note also that the capacity increases logarithmically with the number of receive antennas.

Next, we analyze an important case when both $L N_{t}$ and $N_{r}$ grow large. More specifically, when $L N_{t} \rightarrow \infty$ we need to analyze the two cases based on $N_{t} \rightarrow \infty$ ( $L$ fixed) and $L \rightarrow \infty$ ( $N_{t}$ fixed) separately. Also, we assume $\beta=\frac{N_{r}}{L N_{t}}>1$ and based on this fixed and finite ratio, we examine the ergodic capacity lower bound in the following two cases: 
(i) $L$ : Fixed and $N_{t} \rightarrow \infty, N_{r} \rightarrow \infty$ : In this case, the ergodic capacity lower bound (10) is given by

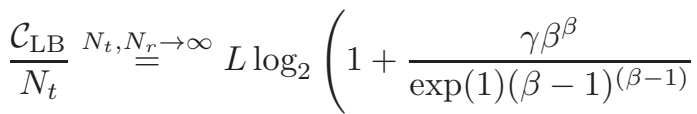

$$
\begin{aligned}
& \left.\times \exp \left(\frac{1}{L} \sum_{i=1}^{L} \ln \mu_{i}-v \ln D_{i}+\exp \left(\frac{2 \lambda_{i}}{\mu_{i}}\right) \operatorname{Ei}\left(\frac{-2 \lambda_{i}}{\mu_{i}}\right)\right)\right) .
\end{aligned}
$$

Proof: Using (26), we express the last sum term in (10) as follows:

$$
\begin{aligned}
\frac{1}{L N_{t}} \sum_{k=0}^{L N_{t}-1} \psi\left(N_{r}\right. & -k) \approx \ln N_{r}+\frac{1}{L N_{t}} \sum_{k=0}^{L N_{t}-1} \ln \left(1-\frac{k}{N_{r}}\right) \\
& \approx \ln N_{r}+\frac{1}{L N_{t}} \int_{0}^{L N_{t}} \ln \left(1-\frac{k}{N_{r}}\right) d k \\
& =\ln N_{r}+(\beta-1) \ln \left(\frac{\beta}{\beta-1}\right)-1
\end{aligned}
$$

where in (29), we expressed the sum term in (28) as an integral function and then, we used the following integral identity [25] to obtain (30)

$$
\int_{0}^{M} \ln \left(1-\frac{x}{N}\right) d x=(N-M) \ln \left(\frac{N}{N-M}\right)-M \text {, if } N>M .
$$

Substituting (30) into (10) gives (27) after simplifications.

From (27), we can infer that the capacity increases linearly with the number of transmit antennas for any $\beta>1$.

(ii) $N_{t}$ : Fixed and $L \rightarrow \infty, N_{r} \rightarrow \infty$ : In this case, we assume that all radio ports are uniformly distributed in a circle of radius $R_{0}$, centered by the base station and all radio ports experience to have the same fading effects. As such, we can set $\mu_{i}=\mu, \lambda_{i}=\lambda$ fixed values and $D_{i}$ varying for all $L$ ports, where $i=1, \ldots, L$. The corresponding probability distribution of the distance between the radio ports and base station is given by $[22$, Eq. (7)]

$$
p_{D}(x)=\frac{2 x}{R_{0}^{2}}, \quad 0 \leq x \leq R_{0}
$$

Next, we rearrange the large-system lower bound in (27) as

$$
\mathcal{C}_{\mathrm{LB}}=L N_{t} \log _{2}\left(1+a \exp \left(\frac{1}{L} \sum_{i=1}^{L} \ln D_{i}^{-v}\right)\right)
$$

where $a \triangleq \gamma \mu \beta^{\beta}(\beta-1)^{(1-\beta)} \exp \left(\exp \left(\frac{2 \lambda}{\mu}\right) \operatorname{Ei}\left(\frac{-2 \lambda}{\mu}\right)-1\right)$. When $L \rightarrow \infty$, the argument in the exponential term is nothing but an ensemble average w.r.t. $D_{i}$ (distribution of distances). As such, we can express (32) as

$$
\frac{\mathcal{C}_{\mathrm{LB}}}{L} \stackrel{L, N_{r} \rightarrow \infty}{=} N_{t} \log _{2}\left(1+a \exp \left(\mathcal{E}_{D_{i}}\left[\ln D_{i}^{-v}\right]\right)\right) \text {. }
$$

We now recall L'Hospital's rule to get

$$
\lim _{x \rightarrow 0^{+}} x \ln (x)=\lim _{x \rightarrow 0^{+}} \frac{\ln (x)}{1 / x}=\lim _{x \rightarrow 0^{+}} \frac{1 / x}{-1 / x^{2}}=0 .
$$

Combining [8, Eq. (2.723.1)] with (34), we can easily obtain the following expression.

$$
\mathcal{E}_{D_{i}}\left[\ln D_{i}^{-v}\right]=-v \ln R_{0}+\frac{v}{2}
$$

Substituting (35) into (33) gives

$$
\frac{\mathcal{C}_{\mathrm{LB}}}{L} \stackrel{L, N_{r} \rightarrow \infty}{=} N_{t} \log _{2}\left(1+a R_{0}^{-v} \exp \left(\frac{v}{2}\right)\right) .
$$

At high-SNRs, we can further approximate (36) as

$$
\frac{\mathcal{C}_{\mathrm{LB}}^{\infty}}{L} \stackrel{L, N_{r} \rightarrow \infty}{=} N_{t} \log _{2}(a)-v N_{t} \log _{2}\left(R_{0}\right)+\frac{v N_{t}}{2 \ln 2} .
$$

From (37), we can clearly infer that a large cell radius $R_{0}$ decreases the ergodic capacity logarithmically.

\section{B. MMSE receivers}

We can now pursue a similar sum rate analysis for the case of MMSE receivers. Assuming independent decoding at the receiver, the achievable sum rate is expressed as [19]

$$
\begin{gathered}
\mathcal{R}^{\mathrm{mmse}}=L N_{t} \mathcal{E}\left[\log _{2}\left(\operatorname{det}\left(\mathbf{I}_{L N_{t}}+\frac{\gamma}{L N_{t}} \mathbf{\Xi}^{H} \mathbf{H}\right)\right)\right] \\
-\sum_{i=1}^{L N_{t}} \mathcal{E}\left[\log _{2}\left(\operatorname{det}\left(\mathbf{I}_{L N_{t}-1}+\frac{\gamma}{L N_{t}} \boldsymbol{\Xi}_{i i} \mathbf{H}_{i}^{H} \mathbf{H}_{i}\right)\right)\right] .
\end{gathered}
$$

Since an exact SNR analysis in tedious, we elaborate on the low- and high-SNR regimes. We begin with the former:

1) Low-SNR analysis: We now examine the sum rate performance of D-MIMO MMSE receivers in the power-limited regime.

Proposition 5: For D-MIMO systems with MMSE receivers in RIG fading channels, the minimum energy per information bit is given by

$$
{\frac{E_{b}}{N_{0 \text { min }}}}^{\text {mmse }}=\frac{\ln 2}{N_{r}\left(N_{t} \sum_{i=1}^{L} \frac{\mu_{i}}{D_{i}^{v}}-\frac{1}{L N_{t}} \sum_{i=1}^{L N_{t}} \sum_{\substack{j=1 \\ j \neq i}}^{L N_{t}} \frac{\mu_{j}}{D_{j}^{v}}\right)}
$$

while the wideband slope is given by (40) shown at the bottom of this page.

Proof: For proving (39), we need to take the first derivative of (38) w.r.t. $\gamma \rightarrow 0$. Using (17), we can easily express the first derivative as

$$
\begin{aligned}
\dot{\mathcal{R}}^{\mathrm{mmse}}(0) & =\frac{L N_{t}}{\ln 2} \mathcal{E}\left[\frac{1}{L N_{t}} \operatorname{tr}\left(\boldsymbol{\Xi} \mathbf{H}^{H} \mathbf{H}\right)\right] \\
& -\frac{1}{\ln 2} \sum_{i=1}^{L N_{t}} \mathcal{E}\left[\frac{1}{L N_{t}} \operatorname{tr}\left(\boldsymbol{\Xi}_{i i} \mathbf{H}_{i}^{H} \mathbf{H}_{i}\right)\right] .
\end{aligned}
$$

$$
\mathcal{S}_{0}^{\mathrm{mmse}}=\frac{2 L N_{t}}{\left.\frac{2 L N_{t}-1}{N_{r}}+L^{2} N_{t} \frac{\sum_{i=1}^{L}\left(\mu_{i}^{2}+\frac{\mu_{i}^{3}}{\lambda_{i}}\right) D_{i}^{-2 v}}{\left(\sum_{i=1}^{L} \mu_{i} D_{i}^{-v}\right)^{2}}-\frac{\left(L N_{t}-1\right)^{2}}{L N_{t}} \sum_{i=1}^{L N_{t}}\left(\frac{\sum_{\substack{L=1 \\ j \neq i}}^{L N_{t}}\left(\mu_{j}^{2}+\frac{\mu_{j}^{3}}{\lambda_{j}}\right) D_{j}^{-2 v}}{\left(\sum_{\substack{L=1 \\ j=i \\ j \neq i}}^{L N_{j}} \mu_{j} D_{j}^{-v}\right.}\right)^{2}\right)} .
$$




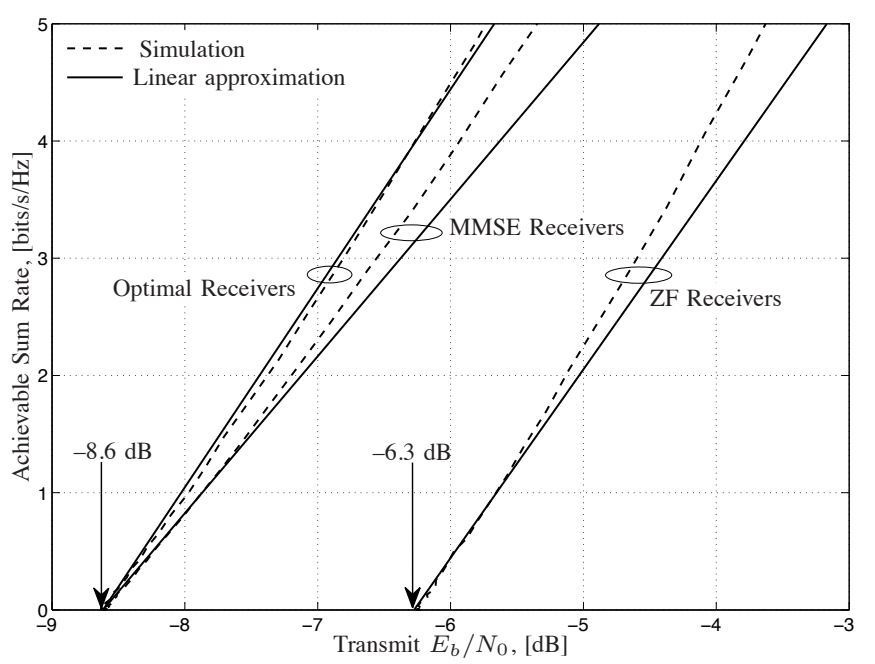

Fig. 3. Low-SNR simulated sum rate (optimal, ZF, and MMSE) and analytical linear approximations against the transmit $E_{b} / N_{0}\left(N_{r}=12\right.$, $N_{t}=2, L=3, \mu_{i}=\mu=1, \lambda_{i}=\lambda=10(\forall i=1, \ldots, L)$, $D_{i}=[1000 \mathrm{~m}, 1500 \mathrm{~m}, 2000 \mathrm{~m}]$, and $\left.v=4\right)$.

Now, we split (41) as $\dot{\mathcal{R}}^{\text {mmse }}(0)=\dot{\mathcal{R}}_{1}^{\text {mmse }}-\dot{\mathcal{R}}_{2}^{\text {mmse }}$. We have already evaluated $\dot{\mathcal{R}}_{1}^{\text {mmse }}$ in (19) and hence, we evaluate $\dot{\mathcal{R}}_{2}^{\text {mmse }}$ as

$$
\dot{\mathcal{R}}_{2}^{\text {mmse }}=\frac{1}{L N_{t} \ln 2} \sum_{i=1}^{L N_{t}} \mathcal{E}\left[\sum_{\substack{j=1 \\ j \neq i}}^{L N_{t}} \frac{\xi_{j}}{D_{j}^{v}} \varphi_{j}\right] .
$$

The expectation is the same as in (18). Therefore, we have that

$$
\dot{\mathcal{R}}^{\text {mmse }}(0)=\frac{N_{r} N_{t}}{\ln 2} \sum_{i=1}^{L} \frac{\mu_{i}}{D_{i}^{v}}-\frac{N_{r}}{L N_{t} \ln 2} \sum_{i=1}^{L N_{t}} \sum_{\substack{j=1 \\ j \neq i}}^{L N_{t}} \frac{\mu_{j}}{D_{j}^{v}} .
$$

To evaluate the wideband slope in (40), we invoke a classical result from random matrix theory on correlated Rayleigh MIMO channels [19, Eq. (75)]. We assume no correlation at the receiver (i.e., $\boldsymbol{\Theta}_{R}=\mathbf{I}_{N_{r}}$ and $\boldsymbol{\Theta}_{T}=\boldsymbol{\Xi}$ ). The dispersion of random matrix $\boldsymbol{\Xi}_{i i}$ denoted by $\zeta\left(\boldsymbol{\Xi}_{i i}\right)$, is given by

$$
\zeta\left(\boldsymbol{\Xi}_{i i}\right)=\frac{\left(L N_{t}-1\right) \sum_{\substack{L=1 \\ j \neq i}}^{L N_{t}}\left(\mu_{j}^{2}+\frac{\mu_{j}^{3}}{\lambda_{j}}\right) D_{j}^{-2 v}}{\left(\sum_{\substack{L=1 \\ j \neq i}}^{L N_{t}} \mu_{j} D_{j}^{-v}\right)^{2}} .
$$

Combining (51) and (52) with [16, Eq. (8)] gives (44). Substituting all the above results into [19, Eq. (75)] and appropriate simplifications give (40).

In Fig. 3, we compare the performance of optimal, linear zero-forcing $(\mathrm{ZF})^{1}$ and MMSE receivers in the low-SNR regime. The simulated low-SNR ergodic capacity/sum rates and the analytical linear approximations are plotted against the transmit $E_{b} / N_{0}$. The figure illustrates the big performance gap between optimal and $\mathrm{ZF}$ receivers which is due to the high number of total transmit antennas $\left(L N_{t}=6\right)$, that corresponds

\footnotetext{
${ }^{1}$ The results for the case of $\mathrm{ZF}$ receivers have been omitted due to space constraints.
}

to the number of interfering data streams. On the other hand, we can easily conclude that the MMSE receivers are optimal in terms of $E_{b} / N_{0 \min }$. MMSE receivers sub-optimality is only reflected via a reduced wideband slope. More importantly, all the analytical linear approximations are sufficiently tight for a wide range of SNR values.

2) High-SNR analysis: In this case, we examine the sum rate performance of MMSE receivers in the high-SNR regime. We recall that, at high-SNRs, both $\mathrm{ZF}$ and MMSE receivers behave equivalently in terms of sum rate [23]. We have already introduced the high-SNR parameters in Section-III-A and hence, we can directly give the following proposition.

Proposition 6: For D-MIMO systems with MMSE receivers in RIG fading channels, the high-SNR parameters are

$$
\begin{aligned}
& \mathcal{S}_{\infty}^{\mathrm{mmse}}=L N_{t} \\
& \mathcal{L}_{\infty}^{\mathrm{mmse}}=\log _{2}\left(L N_{t}\right)-N_{t} \sum_{i=1}^{L}\left(\log _{2}\left(\mu_{i}\right)-v \log _{2}\left(D_{i}\right)\right. \\
& \left.+\frac{1}{\ln 2} \exp \left(\frac{2 \lambda_{i}}{\mu_{i}}\right) \operatorname{Ei}\left(\frac{-2 \lambda_{i}}{\mu_{i}}\right)\right)-\frac{1}{\ln 2} \psi\left(N_{r}-L N_{t}+1\right) \\
& +\frac{1}{L N_{t}} \sum_{i=1}^{L N_{t}} \sum_{\substack{j=1 \\
j \neq i}}^{L N_{t}}\left(\log _{2}\left(\mu_{j}\right)-v \log _{2}\left(D_{j}\right)\right. \\
& \left.+\frac{1}{\ln 2} \exp \left(\frac{2 \lambda_{j}}{\mu_{j}}\right) \operatorname{Ei}\left(\frac{-2 \lambda_{j}}{\mu_{j}}\right)\right)
\end{aligned}
$$

Proof: For MIMO systems with MMSE receivers, the slope and offset parameters are obtained by [19, Eq. (19), (20)] as follows:

$$
\begin{aligned}
\mathcal{S}_{\infty}^{\mathrm{mmse}} & =\min \left(N_{r}, L N_{t}\right) \\
\mathcal{L}_{\infty}^{\mathrm{mmse}} & =\log _{2}\left(L N_{t}\right)-\mathcal{E}\left[\log _{2}\left(\operatorname{det}\left(\boldsymbol{\Xi} \mathbf{H}^{H} \mathbf{H}\right)\right)\right] \\
& +\frac{1}{L N_{t}} \frac{1}{\ln 2} \sum_{i=1}^{L N_{t}} \mathcal{E}\left[\ln \left(\operatorname{det}\left(\boldsymbol{\Xi}_{i i}\right)\right)\right] \\
& +\frac{1}{L N_{t}} \frac{1}{\ln 2} \sum_{i=1}^{L N_{t}} \mathcal{E}\left[\ln \left(\operatorname{det}\left(\mathbf{H}_{i}^{H} \mathbf{H}_{i}\right)\right)\right] .
\end{aligned}
$$

Since $\boldsymbol{\Xi}_{i i}$ is diagonal, we can directly apply (12) to get

$$
\begin{gathered}
\sum_{i=1}^{L N_{t}} \mathcal{E}\left[\ln \left(\operatorname{det}\left(\boldsymbol{\Xi}_{i i}\right)\right)\right]=\sum_{i=1}^{L N_{t}} \sum_{\substack{j=1 \\
j \neq i}}^{L N_{t}}\left(\log _{2}\left(\mu_{j}\right)-v \log _{2}\left(D_{j}\right)\right. \\
\left.+\frac{1}{\ln 2} \exp \left(\frac{2 \lambda_{j}}{\mu_{j}}\right) \operatorname{Ei}\left(\frac{-2 \lambda_{j}}{\mu_{j}}\right)\right)
\end{gathered}
$$

Since $\mathbf{H}_{i}$ is Rayleigh distributed, the term $\mathbf{H}_{i}^{H} \mathbf{H}_{i}$ also follows the central Wishart distribution (zero-mean) [14]. Using [14, Eq. (A.8.1)], the last expectation term in (48) can be easily evaluated. Then, substituting (11), (49) into (48), and appropriate simplifications give (46). 
Similar to optimal receivers, the small- and large-scale fading terms are decoupled in the high-SNR regime, while (45) verifies that the high-SNR sum rate increases linearly with the minimum number of antennas. As anticipated, higher Tx-Rx distances reduce the sum rate due to the increased path-loss attenuation.

\section{CONCLUSION}

In this paper, we have presented an analytical framework to investigate the performance of D-MIMO systems with optimal and linear MMSE receivers operating over RIG fading channels. More specifically, the implications of small- and large-scale fading effects were analyzed in detail. The main motivation for our performance analysis has been the poor accuracy of the gamma distribution to approximate the lognormal distribution, when the variance of the latter is large. As such, the RIG distribution can serve as an efficient approximation to the classical Rayleigh/log-normal model. At the same time, the mathematical analysis becomes more challenging.

We derived new closed-form upper and lower capacity bounds for this class of channels, which apply for any arbitrary number of antennas and remain sufficiently tight across the entire SNR range. In the high-SNR regime, we explicitly demonstrated that the lower bound becomes exact and in the low-SNR regime, we derived new analytical expressions for the minimum energy per information bit to reliably convey any positive rate and the wideband slope.

\section{APPENDIX}

Lemma 1: For IG distributed RV, $\xi_{i} \sim \operatorname{IG}\left(\mu_{i}, \lambda_{i}\right)$, the first log-moment is given by

$$
\mathcal{E}\left[\ln \xi_{i}\right]=\ln \mu_{i}+\exp \left(\frac{2 \lambda_{i}}{\mu_{i}}\right) \operatorname{Ei}\left(\frac{-2 \lambda_{i}}{\mu_{i}}\right)
$$

where $i=1, \ldots, L$. The following key results will be particularly useful to obtain the dispersion of large-scale fading random matrix $\Xi$.

$$
\begin{aligned}
& \mathcal{E}[\operatorname{tr}(\boldsymbol{\Xi})]=N_{t} \sum_{i=1}^{L} \mathcal{E}\left[\xi_{i}\right] D_{i}^{-v}=N_{t} \sum_{i=1}^{L} \mu_{i} D_{i}^{-v} \\
& \mathcal{E}\left[\operatorname{tr}\left(\boldsymbol{\Xi}^{2}\right)\right]=N_{t} \sum_{i=1}^{L} \frac{\mathcal{E}\left[\xi_{i}^{2}\right]}{D_{i}^{2 v}}=N_{t} \sum_{i=1}^{L}\left(\mu_{i}^{2}+\frac{\mu_{i}^{3}}{\lambda_{i}}\right) D_{i}^{-2 v} .
\end{aligned}
$$

Proof: Using (2) and the integral identity [24, Eq. (2.6.22.8)], we can easily obtain (50) after basic simplifications. We now recall that for IG distributed RVs, the first two moments about zero are $\mathcal{E}\left[\xi_{i}\right]=\mu_{i}$ and $\mathcal{E}\left[\xi_{i}^{2}\right]=\mu_{i}^{2}+\frac{\mu_{i}^{3}}{\lambda_{i}}$, respectively [9]. Using these two moments, we can directly obtain (51) and (52), respectively.

\section{ACKNOWLEDGMENTS}

The work of M. Matthaiou has been supported in part by the Swedish Governmental Agency for Innovation Systems (VINNOVA) within the VINN Excellence Center Chase. The work of C. Zhong has been supported by the Fundamental
Research Funds for Central Universities (2012QNA5011) and the Zhejiang Provincial Natural Science Foundation of China (No. LQ12F01006).

\section{REFERENCES}

[1] H. Zhang and H. Dai, "On the capacity of distributed MIMO systems," in Proc. Conf. Inform. Sciences and Systems (CISS), Princeton University, Princeton, NJ, Mar. 2004.

[2] C. Zhong, K.-K. Wong, and S. Jin, "Capacity bounds for MIMO Nakagami- $m$ fading channels," IEEE Trans. Signal Process., vol. 57, no. 9, pp. 3613-3623, Sep. 2009.

[3] M. Matthaiou, N. D. Chatzidiamantis, G. K. Karagiannidis, and J. A. Nossek, "On the capacity of generalized- $K$ fading MIMO channels," IEEE Trans. Signal Process., vol. 58, no. 11, pp. 5939-5944, Nov. 2010.

[4] Karmeshu and R. Agrawal, "On efficacy of Rayleigh-Inverse Gaussian distribution over K-distribution for wireless fading channels," Wireless Commun. Mob. Comput., vol. 7, no. 1, pp. 1-7, Jan. 2007.

[5] N. D. Chatzidiamantis, H. G. Sandalidis, G. K. Karagiannidis, and M. Matthaiou, "Inverse Gaussian modeling of turbulence-induced fading in free-space optical systems," J. Lightw. Technol., vol. 29, no. 10, pp. 1590 1596, May 2011.

[6] A. Laourine, M.-S. Alouini, S. Affes, and A. Stéphenne, "On the performance analysis of composite multipath/shadowing channels using the $\mathcal{G}$-distribution,' IEEE Trans. Commun., vol. 57, no. 4, pp. 1162-1170, Apr. 2009.

[7] C. Zhong, M. Matthaiou, G. K. Karagiannidis, A. Huang, and Z. Zhang, "Capacity bounds for AF dual-hop relaying in $\mathcal{G}$ fading channels," IEEE Trans. Veh. Technol., vol. 61, no. 4, pp. 1730-1740, May 2012.

[8] I. S. Gradshteyn and I. M. Ryzhik, Table of Integrals, Series, and Products, 7th ed., San Diego, CA: Academic, 2007.

[9] R. S. Chhikara and J. L. Folks, The Inverse Gaussian Distribution: Theory, Methodology, and Applications, New York: Marcel Dekker, 1989.

[10] M. Evans, N. Hastings, and B. Peacock, Statistical Distributions, 3rd ed., New York: Wiley, 2000.

[11] M. Abramowitz and I. A. Stegun, Handbook of Mathematical Functions with Formulas, Graphs, and Mathematical Tables, 9th ed., New York: Dover, 1970.

[12] A. P. Prudnikov, Y. A. Brychkov, and O. I. Marichev, Integrals and Series, Vol. 3: More Special Functions, New York: Gordon \& Breach, 1990

[13] Ö. Oyman, R. Nabar, H. Bölcskei, and A. Paulraj, "Characterizing the statistical properties of mutual information in MIMO channels," IEEE Trans. Signal Process., vol. 51, no. 11, pp. 2782-2795, Nov. 2003.

[14] A. Grant, "Rayleigh fading multi-antenna channels," EURASIP J. Appl. Signal Process., vol. 2002, no. 3, pp. 316-329, Mar. 2002.

[15] S. Verdú, "Spectral efficiency in the wideband regime," IEEE Trans. Inf. Theory, vol. 48, no. 6, pp. 1319-1343, Jun. 2001.

[16] A. Lozano, A. M. Tulino, and S. Verdú, "Multiple-antenna capacity in the low-power regime," IEEE Trans. Inf. Theory, vol. 49, no. 10, pp. 2527-2544, Oct. 2003.

[17] S. Shamai (Shitz) and S. Verdú, "The impact of frequency-flat fading on the spectral efficiency of CDMA," IEEE Trans. Inf. Theory, vol. 47, no. 4, pp. 1302-1327, May 2001.

[18] A. Lozano, A. M. Tulino, and S. Verdú, "High-SNR power offset in multiantenna communications," IEEE Trans. Inf. Theory, vol. 51, no. 12, pp. 4134-4151, Dec. 2005.

[19] M. R. McKay, I. B. Collings, and A. M. Tulino, "Achievable sum rate of MIMO MMSE receivers: A general analytic framework," IEEE Trans. Inf. Theory, vol. 56, no. 1, pp. 396-410, Jan. 2010.

[20] M. Matthaiou, N. D. Chatzidiamantis, and G. K. Karagiannidis, "A new lower bound on the ergodic capacity of distributed MIMO systems," IEEE Signal Process. Lett., vol. 18, no. 4, pp. 227-230, Apr. 2011.

[21] F. Rusek, et al., "Scaling up MIMO: Opportunities and challenges with very large arrays," in press IEEE Signal Process. Mag., 2012.

[22] C. T. Lau and C. Leung, "Capture models for mobile packet radio networks," IEEE Trans. Commun., vol. 40, no. 5, pp. 917-925, May 1992.

[23] A. Paulraj, R. Nabar, and D. A. Gore, Introduction to Space-Time Wireless Communications, Cambridge University Press, May 2003.

[24] A. P. Prudnikov, Y. A. Brychkov, and O. I. Marichev, Integrals and Series, Vol. 1: Elementary Functions, New York: Gordon \& Breach, 1986.

[25] Wolfram, The Wolfram|Alpha Site [Online]. Available: http://www.wolframalpha.com/ 